\title{
Роль операции продольной резекции желудка в обеспечении гликемического контроля у пациентов с сахарным диабетом 2-го типа и ожирением
}

\author{
Т. Омаров, С. Салахова, А. Ибрагимова, Н. Байрамов \\ Азербайджанский медицинский университет, г. Баку
}

\section{Role of operation of longitudinal gastric resection in guaranteeing of glycemic control in patients, suffering diabetes mellitus type II and obesity}

\author{
T. Omarov, S. Salahova, A. Ibrahimova, N. Bayramov \\ Azerbaijani Medical University, Baku, Azerbaijani Republic
}

\section{Реферат}

Цель. Изучить корреляцию между контролем уровня гликемии и степенью снижения индекса массы тела у пациентов после бариатрической операции.

Материалы и методы. Проведен контроль уровня обмена глюкозы у 84 пациентов с предиабетом и сахарным диабетом 2-го типа, страдающих патологическим ожирением, после операции уменьшения желудка.

Результаты. Из 84 пациентов у 30 (35,7\%) продолжительность заболевания сахарным диабетом 2-го типа составляла 1 год, у 27 (32,1\%) - 10 лет, у 12 (14,3\%) - более 10 лет. У 15 (17,9\%) пациентов диагностирован предиабет. При изучении антропометрических показателей отмечено снижение массы тела на $38 \%$ после операции по сравнению с исходными показателями. Индекс массы тела (ИМТ) снизился с 46,8 (41,3 - 54,3) до 27,6 (25,4 - 30,9) кг/м² (p=0,001). Через 2 года после операции общее снижение массы тела по сравнению с исходными показателями составило 42\%, а ИМТ снизился до 26,8 $(23,1-28,4)$ кг/м². Ремиссия сахарного диабета 2-го типа в сроки наблюдения 1 - 3 года отмечена у 82 (98,6\%) пациентов (уровень гликированного гемоглобина менее 6,0\%, глюкозы натощак менее 5,6 ммоль/л).

Выводы. Результаты проведенного исследования показали, что продольная резекция желудка у больных с сахарным диабетом 2-го типа эффективна не только для уменьшения ИМТ, но и для коррекции метаболического синдрома. В послеоперационном периоде у них наблюдали стабильность показателей гликемии и углеводного обмена.

ключевые слова: чрезмерное ожирение; рукавная гастрэктомия; шунтирование желудка; сахарный диабет 2-го типа; бариатрическая хирургия.

Abstract

Objective. To study a correlation between control of the glycaemia level and degree of the body mass index (BMI) in patients after bariatric operation.

Materials and methods. Control of the glucose metabolism level was conducted in 84 patients, suffering prediabetes and diabetes mellitus type II, morbid obesity after the gastric reduction operation.

Results. In 30/84 (35.7\%) patients the diabetes mellitus type II duration have constituted 1 year, in 27 (32.1\%) - 10 years, and in $12(14.3 \%)$ - more than 10 years. In $15(17.9 \%)$ patients a prediabetes was diagnosed. While studying of anthropometric indices a BMI lowering by 38\% postoperatively, comparing with initial indices, was noted. The BMI have lowered from 46.8 (41.3 - 54.3) to $27.6(25.4-30.9) \mathrm{kg} / \mathrm{m}^{2}(\mathrm{p}=0.001)$. In 2 years postoperatively a general lowering of the body mass, comparing with primary indices, have constituted $42 \%$, and BMI have lowered to $26.8(23.1-28.4) \mathrm{kg} / \mathrm{m}^{2}$. Remission of diabetes mellitus type II in terms of observation 1 - 3 years was noted in 82 (98.6\%) patients (level of glycaemized hemoglobin lesser than $6.0 \%$, glucose in fasting state lesser than $5,6 \mathrm{mmol} / \mathrm{l})$.

Conclusion. Results of the investigation conducted have shown, that longitudinal gastric resection in patients, suffering diabetes mellitus type II is effective not only for the BMI lowering, but for the metabolic syndrome as well. In postoperative period in them a stability of the glycaemia and carbohydrate metabolism indices was followed.

Keywords: morbid obesity; sleeve gastrectomy; gastric shunting; type II diabetes mellitus; bariatric surgery.

Исследования, проведенные в последние годы, показывают, что достаточно большое число больных с сахарным диабетом (СД) 2-го типа имеют ожирение. Если в 2015 г. таких больных было 415 миллионов, то к 2040 г. прогнозируется увеличение их числа до 642 миллионов. После бариатрической операции по поводу указанной патологии у этих больных прогрессируют метаболические изменения, в частности инсулинрезистентность, что приводит к неудовлетворительным результатам лечения . Считают, что лечение ожирения и СД 2-го типа, наряду с фармакотерапией, должно включать изменение образа жизни больного. По данным проведенных статистических исследований ожирение среди населения приобретает характер эпидемии. Всемирная организация здравоохранения приводит следующие данные: в 2011 г. зарегистрировано 2,1 миллиарда больных с метаболическим синдромом и ожирением, к 2025 г. ожидается увеличение числа таких больных в 2 раза. Хотя в нашей стране нет точных 
статистических данных в отношении указанной патологии, но больных с ожирением достаточно много [1 - 3].

Эффективность консервативного лечения ожирения или морбидного ожирения не превышает 5 - 10\%. На основании клинического опыта разные авторы придерживаются единого мнения: снизить массу тела (МТ) у больных с ожирением и СД 2-го типа очень сложно. Несмотря на многообразие препаратов для коррекции уровня сахара, только у 40\% больных возможен гликемический контроль $[4,5]$.

В последние десятилетия во всем мире возрастает интерес к хирургическому лечению ожирения. По данным Оздоровительного центра Соединенных Штатов Америки у 60\% больных с ожирением после диеты и физической нагрузки сниженная МТ держится только в течение года. Через 5 лет первоначальная МТ возвращается почти у всех больных [6].

Одни исследователи считают, что основным показанием к хирургическому лечению, наряду с выраженным повышением МТ, является воздействие на заболевания, связанные с ожирением, другие - что достижение контроля гликемии, а также липидного обмена дает более эффективные возможности для коррекции СД 2-го типа и связанных с ним осложнений [7, 8].

Различные авторы после бариатрических операций, выполненных пациентам с метаболическими изменениями, наблюдали компенсацию СД 2-го типа на фоне основного заболевания. Это послужило основанием для введения отдельного нозологического термина «метаболическая хирургия». Устойчивая компенсация СД 2-го типа в этом направлении была подтверждена исследованиями F. Li и соавторов [9]. Поэтому важно изучать изменения углеводного метаболизма после бариатрических операций для улучшения результатов лечения пациентов с ожирением и СД 2-го типа. Учитывая изложенное, мы также решили изучить корреляционную связь между гликемическим контролем, регуляцией углеводного обмена и понижением МТ после бариатрической операции у пациентов с ожирением и СД 2-го типа. Изучение влияния бариатрической операции на углеводный метаболизм у больных с СД 2-го типа и чрезмерным ожирением очень важно для медицинской практики.

Цель исследования: изучение результатов лечения больных с СД 2-го типа и чрезмерным ожирением, которым выполнена продольная резекция желудка.

\section{Материалы и методы исследования}

Проведен ретроспективный и проспективный анализ состояния обмена глюкозы в послеоперационном периоде у 84 больных с предиабетом и СД 2-го типа и чрезмерным ожирением, оперированных в клинике "Modern Hospital”. Мужчин было 25 (29,8\%), женщин - 59 (70,2\%). Возраст пациентов колебался от 28 до 60 лет, средний возраст составил $(46 \pm 1,6)$ года. В возрасте младше 40 лет были 22 (26,2\%) больных, старше 40 лет - 62 (73,8\%) больных.

МТ составляла 137,0 (118 - 162) кг, ИМТ - 35,0 - 68,0 кг/ $\mathrm{M}^{2}$, в среднем $(47,9 \pm 2,3)$ кг $/ \mathrm{M}^{2}$. У $48(57,1 \%)$ пациентов до операции в анамнезе отмечена артериальная гипертензия. У 43 (59,7\%) из 72 (85,7\%) больных до операции ИМТ был в пределах 40,0 - 49,9 кг/м², у 24 (33,3\%) - 50,0 - 59,9

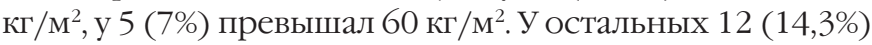
больных наблюдали II степень ожирения.

У 38 (45,2\%) больных диагностирован предиабет. У 34 (40,5\%) больных продолжительность заболевания СД 2го типа составила 1 год, у 12 (14,3\%) - от 1 до 10 лет. До операции 59 (70,2\%) больных с ожирением принимали пероральные препараты для лечения СД, 10 (11,9\%) пациентов использовали инсулин. Не получали никаких лекарств 15 (17,9\%) больных с предиабетом. Средний возраст этих больных составил $(46 \pm 2,8)$ года, у них серьезных осложнений СД не выявлено. Уровень гликированного гемоглобина (HbA1c) в крови составлял 7\%, глюкозы натощак - 7 ммоль/л, общего холестерина - 4,5 ммоль/л, липопротеинов высокой плотности - 2,6 ммоль/л.

У болышинства больных диагностирована хроническая декомпенсация метаболизма глюкозы. Первоначально из 59 пациентов, которые не получали инсулинотерапию, у большинства выявлена острая гиперинсулинемия. Кроме того, у них была резистентность к инсулину. Таким образом, хотя большинству больных с СД 2-го типа и ожирением до бариатрической операции проводили консервативное лечение, метаболический уровень углеводного обмена (выше 60\%) был недостаточным для достижения удовлетворительных результатов.

\section{Результаты}

Изучение динамики антропометрических данных показало, что после операции у наблюдаемых больных МТ снизилась на 38\% по сравнению с исходными показателями, а ИМТ - с 46,8 (41,3 - 54,3) до 27,6 (25,4 - 30,9) кг / $\mathrm{M}^{2}(\mathrm{p}=0,001)$.

Через 2 года после операции МТ снизилась на 42\% по сравнению с исходными показателями, а ИМТ - до 26,8 $(23,1-28,4)$ кг м² $^{2}$ По сравнению с дооперационным показателем ИМТ в течение 3 лет после бариатрической операции стабильно снижался $(\mathrm{p}=0,05)$. Через 3 года после операции у больных ожирения не отмечено, но на 5-м году у 2 (2,4\%) больных наблюдали ожирение II степени, у 5 (6\%) - І степени, у 8 (9,5\%) - полноту. У 69 (82,1\%) больных была нормальная МТ.

Принимая во внимание приведенные данные, мы считаем, что изучение патогенетических изменений, направленных на предотвращение развития метаболического синдрома после бариатрической операции у пациентов с предиабетом и СД 2-го типа, а также корреляции между ними, является многообещающим в аспекте улучшения результатов лечения.

Таким образом, результаты ретроспективного анализа подтвердили, что операции уменьшения желудка эффективны для снижения ИМТ. Положительная динамика ИМТ была более существенной в первые 2 года после операции. Изучение состояния обмена глюкозы после продольной резекции желудка позволяет сделать вывод о том, что гликемический контроль после предупреждения гипогликемии был достигнут. Инсулинотерапию последовательно прекращали в первые дни после операции. Через 3 мес после операции прекратили принимать пероральные сахароподдерживающие препараты 1,4\% больных. 
Изучая гликемию натощак, статистически достоверную динамику мы отметили через 3 мес после операции - в среднем 5,6 (4,5 - 6,3) ммоль/л (р > 0,001). Тенденцию снижения уровня сахара при последующих контрольных обследованиях наблюдали через 6 мес - 5,1 (4,2 $5,8)$ ммоль/л. Показатели гликемии натощак достоверно отличались через 3 и 6 мес после операции $(\mathrm{p}=0,79)$. По результатам лабораторных и клинических исследований в послеоперационном периоде ни у одного больного не обнаружено гипогликемии. В сроки наблюдения 1 и 1,5 года после операции уровень глюкозы в крови составлял 3,0 ммоль/л у пациентов, которые не получали пероральных сахаропонижающих препаратов, у них никаких клинических симптомов не выявлено.

Через 1 мес после операции число больных, у которых гликемия натощак не превышала 7 ммоль/л, из 27 (32\%) увеличилось до 63 (75\%), через 3 мес - до 66 (78\%). Через 9 мес у всех 84 (100\%) больных наблюдали указанный уровень гликемии.

\section{Обсуждение}

Таким образом, у больных, которые получали инсулинотерапию до операции, в послеоперационном периоде уровень сахара был нормализован, несмотря на постепенное прекращение приема инсулина в течение 1 мес. Уровень НbA1с составил 1,8\% в первый месяц после операции, 2,9\% - через 3 мес и 7,0\% - через 1 год.

В послеоперационном периоде стабилизировалась артериальная гипертензия. У большинства пациентов дозу гипотензивных препаратов снизили.

Неполную ремиссию диабета 2-го типа (уровень HbA1c менее 6,55\%, гликемия натощак менее 7,0 ммоль/л) отметили у 82 (98,6\%) пациентов в сроки наблюдения от 1 года до 5 лет.

\section{Выводы}

1. Результаты исследования показали, что продольная резекция желудка у больных с СД 2-го типа эффективна не только для уменьшения ИМТ, но и для коррекции метаболического синдрома. Это было доказано стабильностью гликемического контроля, а также углеводного обмена в послеоперационном периоде.

2. У больных с ожирением и СД 2-го типа бариатрические операции являются перспективным направлением для предотвращения возникновения метаболического синдрома и генетических проблем.

\section{References}

1. Hayes K, Eid G. Laparoscopic Sleeve Gastrectomy: Surgical Technique and Perioperative Care. Surg Clin North Am. 2016 Aug;96(4):763-71. doi:10.1016/j.suc.2016.03.015.

2. Golomb I, Ben David M, Glass A, Kolitz T, Keidar A. Long-Term Metabolic Effects of Labaroscopic Sleeve Gastrectomy. JAMA Surg. 2015 Nov;150(11):1051-7. doi:10.1001/jamasurg.2015.2202

3. Buchwald H, Estok R, Fahrbach K. Weight and type 2 diabetes after bariatric surgery: systemic review and meta-analysis. Am J Med. 2009 Mar;122(3):248-56. doi:10.1016/j.amjmed.2008.09.041.

4. Collier A. Bariatric surgery and diabetes. Indian J Endocrinol Metab. 2012 Dec;16(Suppl 2):S230-2. doi: 10.15406/jdmdc.2017.04.00110.

5. Demaria E, Winegar D, Pate V. Early postoperative outcomes of metabolic surgery to treat diabetes from sites participating in the ASMBS bariatric surgery center of excellence program as reported in the Bariatric Outcomes Longitudinal Database. Ann Surg. 2010 Sept; 252(3):559-66. doi:10.1097/SLA.0b013e3181f2aed0.

6. Dixon JB, Browne JL, Lambert GW, Jones KM, Reddy P, Pouwer F, Speight J. Severely obese people with diabetes experience impaired emotional well-being associated with socioeconomic disadvantage: results from diabetes MILES-Australia. Diabetes Res Clin Pract. 2013 Aug; 101(2):131-40. doi:10.1016/j.diabres.2013.05.017

7. Fried M, Yumuk V, Oppert J, Scopinaro N. Interdisciplinary European guidelines on metabolic and bariatric surgery/International Federation for the Surgery of Obesity and Metabolic Disorders-European Chapter(IFSOEC) and European Association for the Study of Obesity. Obes Surg. 2014 Jan;24(1):42-55. doi: 10.1007/s11695-013-1079-8.

8. Hayes K, Eid G. Laparoscopic Sleeve Gastrectomy: Surgical Technique and Perioperative Care. Surg Clin North Am. 2016 Aug;96(4):763-71. doi:10.1016/j.suc.2016.03.015.

9. Li F, Peng Y, Zhang M. Sleeve gastrectomy activates the GLP-1 pathway in pancreatic $\beta$ cells and promotes GLP-1-expressing cells differentiation in the intestinal tract. Mol. Cell Endocrinol. 2016 Nov 15; 436:33-40. doi:10.1016/j.mce.2016.07.019. 